\title{
A component analysis of the generation and release of isometric force in Parkinson's disease
}

\author{
Nigel Jordan, Harvey J Sagar, James A Cooper
}

\begin{abstract}
Paradigms of isometric force control allow study of the generation and release of movement in the absence of complications due to disordered visuomotor coordination. The onset and release of isometric force in Parkinson's disease (PD) was studied,using computerised determinants of latency of response and rate of force generation and release. Components of isometric force control were related to measures of cognitive, affective and clinical motor disability. The effects of treatment were determined by longitudinal study of de novo patients. Patients with PD showed impairment in latency and rate of force change for movement release as well as onset. Rate of force change correlated with depression, clinical motor disability and memory quotient but latency showed no correlation with any of these measures. Treatment improved rate of force release, in concert with clinical motor disability, but not latency. These results suggest dissociations between latency and rate of force change that may be linked to different neurochemical deficits. Further, they demonstrate akinetic deficits in force release that argue against the "neural energy hypothesis" of akinesia.
\end{abstract}

Parkinson's disease (PD) is, primarily, a disorder of voluntary movement, but cognitive deficits and depression are also recognised as common features. ${ }^{12}$ Clinical observations of PD have stressed akinesia as the key component of the motor disorder which, with rigidity and tremor, forms the classical clinical triad. Most research on disordered movement in PD has been directed at the isotonic profile; in isotonic tasks, an overt movement is required for successful response, for example, to track a moving target, ${ }^{3}$ or to combine or sequence set movements. ${ }^{45}$ The nature of the isometric force profile in PD is, however, also important for several reasons: first, clinical complaints of weakness are common; second, isometric tasks, in contrast to isotonic tasks, do not involve visual perception and spatial orientation which may be separately affected by cognitive disturbance; third, isometric movement (for example, squeeze) has been proposed as the best correlate of akinesia; ${ }^{5}$ fourth, the isometric force profile allows clearer evaluation of force relaxation as well as production, an important area in view of the clinical observations that PD patients show particular difficulty in arresting movement.

Interpretation of studies on motor control in PD is complicated by several clinical factors which confound motor performance. First, disease chronicity alters the pattern of motor deficit in that treatment failure and involuntary movements are particular features of the later stages of the disease. Second, the duration and nature of treatment at the time of assessment may have variable influences on motor control. For example, dyskinesia is a common complication of long term levodopa therapy but is less common with dopamine agonists and probably absent with anticholinergic treatment alone. Third, coexistent cognitive deficits and depression may influence the performance of patients with PD on some motor measures, notably reaction time, ${ }^{6}$ and may influence more complex motor tasks. Seldom has control of all these clinical factors been incorporated in a single experimental design to study motor control in PD.

In the light of these experimental and clinical considerations, we undertook a study of PD using isometric force as a purer measure of motor control than isotonic movement. We aimed to establish a component analysis of isometric force generation through latency to force onset, rate of force generation, maximum force, latency to force release and rate of force release. Maximal force was chosen to obviate the need for cognitive estimation or the compounding factors of visual feedback and perceptual effects. Other specific aims were to evaluate the isometric force profile in PD as a quantitative measure of akinesia; to relate component measures of movement, notably reaction time and force change to cognitive and affective variables; and to determine the effects of treatment on these component measures.

\section{Methods}

SUBJECTS

The subject groups comprised thirty two patients with newly diagnosed, untreated PD, twenty nine patients with PD currently on treatment and twenty four healthy control subjects (table 1). Newly diagnosed patients were drawn from consecutive referrals to the department of neurology. The diagnosis was based on the presence of akinesia plus rigidity, rest tremor or postural instability and absence of clinical signs of other causes of Parkinsonism. Treated PD patients were willing participants drawn from the outpatient clinics of the 
Table 1 Characteristics of subject groups. Figures represent means with SEM in brackets.

\begin{tabular}{lllllll}
\hline Group & M:F & Age & $\begin{array}{l}\text { Disease } \\
\text { duration } \\
\text { (years) }\end{array}$ & $\begin{array}{l}\text { Education } \\
\text { (years) }\end{array}$ & $\begin{array}{l}\text { NART } \\
I Q\end{array}$ & $\begin{array}{l}\text { KCRS } \\
\text { score }\end{array}$ \\
\hline Healthy control & $13: 11$ & $58 \cdot 6(4 \cdot 2)$ & NA & $10 \cdot 3(0 \cdot 7)$ & $108(6 \cdot 7)$ & NA \\
Parkinson & $36: 25$ & $59 \cdot 0(4 \cdot 1)$ & $1 \cdot 2(1 \cdot 8)$ & $9 \cdot 7(0 \cdot 5)$ & $109(6 \cdot 4)$ & $18(1 \cdot 7)$ \\
\hline
\end{tabular}

neurology department. The subject groups did not differ significantly in age, sex or IQ. The untreated and treated PD subgroups differed significantly in disease duration but not motor disability. Thus more advanced disease in the treated PD group was masked by treatment; of importance for the purposes of this experiment, the groups were matched in clinical motor disability.

Medication in the treated group comprised a levodopa preparation (11 patients, mean dose $200 \mathrm{mg} /$ day, range 100 to $500 \mathrm{mg} /$ day), bromocriptine ( 9 patients, mean dose $15 \mathrm{mg} /$ day, range 4 to $35 \mathrm{mg} /$ day), and anticholinergic therapy with benzhexol ( 6 patients, mean dose $8 \mathrm{mg} /$ day, range 2 to $30 \mathrm{mg} /$ day). Three patients were receiving polypharmacy usually a levodopa preparation plus an anticholinergic.

Twenty one of the untreated patients were reassessed after stabilisation of monotherapy with levodopa, bromocriptine or benzhexol. Treatment produced a significant improvement in clinical motor disability, $(p<0.01)$ as measured by score on the King's College Rating Scale (KCRS) a detailed quantitative measure of clinical symptoms and signs. ${ }^{12}$ Fourteen of the control subjects were reassessed at a similar interval.

None of the subjects had a past history of head injury, alcohol abuse or other neurological or general medical conditions that may produce motor or cognitive impairment. No subject was receiving psychoactive medication. None of the untreated patients had received levodopa or bromocriptine at any time before the study.

PROCEDURE

The memory and orientation section of the Blessed Dementia Scale (BDS) ${ }^{7}$ was used to quantify the degree of overall cognitive impairment. Intelligence quotient (IQ) was estimated using the National Adult Reading Test. ${ }^{8} \mathrm{Mem}$ ory was assessed using the Wechsler Memory Scale $^{9}$ which provides a memory quotient (MQ) parallel to IQ in intelligence. Frontal lobe function was assessed from the number of categories achieved in the Wisconsin Card Sorting Test (WCST). ${ }^{10}$ Affective disturbance was quantified by the Beck Depression Inventory (BDI). ${ }^{11}$ Overall motor disability was assessed using the Kings College Rating Scale (KCRS), a detailed quantitative measure of all aspects of motor disability including separate measures of rigidity, tremor, akinesia and the performance of activities of daily living. ${ }^{12}$ Finger dexterity, Fine Finger Movements (FFM), was evaluated from the ability of subjects to rotate a spindle between the thumb and forefinger of each hand; results were expressed as the number of revolutions achieved in thirty seconds, averaged across the two hands.

FORCE CONTROL

Isometric force was measured using a hand grip dynamometer which consisted of a manipulandum of two aluminium strips padded with foam. The strips were connected to a sturdy connecting frame and strain measuring device. The apparatus was fixed with clamps to a sturdy shelf which could be altered in height and position so that the manipulandum could be gripped easily with the hand. This device has been reported to give a reproducible and acceptable measure of hand grip strength. ${ }^{13}$ Output from the device was converted into a direct measure of applied force in Newtons (N) by a BBC Master Series microcomputer. New computer programs were written to enable the measurement of both reaction time and rate of change of force for each isometric movement.

During the experiment, the subjects were seated with the manipulandum positioned to one side, such that the device could be easily and comfortably gripped. Instructions were given to the subject to grip the device as hard and as quickly as possible on hearing a $250 \mathrm{~ms}$ auditory tone, delivered by the computer, and to maintain the force until a second tone was heard, four seconds later; subjects were then to release force as quickly as possible. The output of force applied to the handgrip was sampled every 0.03 seconds throughout the experiment. The time from the first tone until an increment of ten Newtons had been reached was recorded as reaction time to force onset. Rate of acquisition of maximal force was measured as the rate of force applied until three consecutive 0.03 second interval force recordings had been within ten Newtons of each other, implying the flattening of the curve as maximal force was reached. Once three such recordings were achieved, the force applied was divided by the time taken to reach the second of the three recordings to give a measure of mean rate of acquisition of maximal force in Newtons per second. Reaction time to force release was taken as the interval between the second tone and release of force through ten Newtons. The rate of release of force was calculated from the absolute force level at the end of the reaction time to force release divided by the time taken to reduce the force level to zero Newtons, expressed as Newtons per second.

Subjects were given three practice attempts. Trials consisted of two blocks of five attempts, separated by a fifteen minute rest period, and repeated for both hands.

\section{STATISTICAL ANALYSIS}

Measures of isometric force control are expressed as reaction time to force onset, reaction time to force release, maximum force, rate of force generation and rate of force relaxation. The data set for each variable in the total PD group was assessed for normality of distribution using the Kolmogarof-Smirnov 
Figure 1 Maximum grip in Newtons (mean and SEM). The two PD groups and healthy control subjects did not differ from each other.
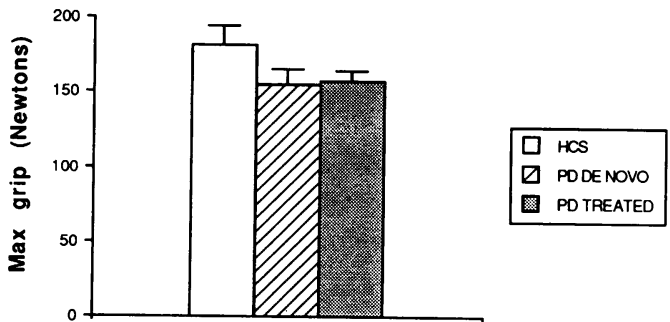

test. Some data sets were found not to be normally distributed; therefore non-parametric statistics were used throughout. Paired comparisons were made between data sets using Mann-Whitney $U$ tests. Correlations between data sets were evaluated using the Spearman rank correlation coeffident, rho. Assessment of change in those patients who were assessed before and after treatment was made using the Wilcoxon Paired Signed Ranks Test. All paired comparisons were two tailed unless otherwise stated.

\section{Results}

MAXIMUM GRIP (FIGURE 1)

The three subject groups, patients before treatment, medicated patients and control subjects did not differ significantly in maximum grip. Thus differences between the groups in reaction time or rates of changes of force cannot be attributed to differences in strength.

ISOMETRIC REACTION TIME (FIGURE 2)

Untreated and treated PD groups differed from the control group in latency, that is, all two scores to two decimal places, to force onset $(\mathrm{z}=3.69, \mathrm{p}<0.001$ and $\mathrm{z}=3.33, \mathrm{p}<0.001$ respectively) and release $(z=4 \cdot 19, p<0.0001$ and $z=3.67, p<0.001$ respectively) but the untreated and treated patients did not differ significantly from each other.

FORCE GENERATION AND RELEASE (FIGURE 3)

Compared with control subjects the rate of force generation was significantly slowed in the untreated as well as the treated patients $(\mathrm{z}=3.96, \mathrm{p}<0.0001$ and $\mathrm{z}=3.59, \mathrm{p}<0.001$ respectively); parallel slowing was seen in the rate of release of force in both patient groups (untreated $\mathrm{z}=3.59, \mathrm{p}<0.001 ;$ treated

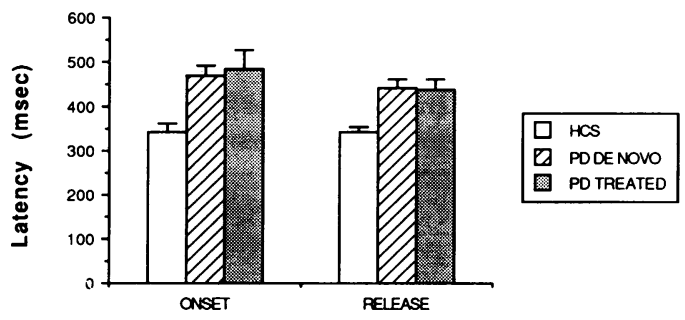

Figure 2 Latency to onset and release of isometric force in groups of untreated and treated PD patients, matched for motor disability. Results expressed as mean and SEM. The two PD groups were impaired in latency to onset and The two PD groups were impaired in latency to onset and
release of isometric force but did not differ from each other.

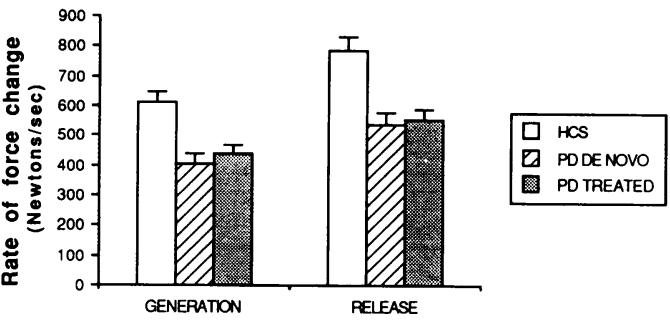

Figure 3 Rate of change of force during generation and release of maximum isometric force in groups of untreated and treated PD patients, matched for motor disability.

Results expressed as mean and SEM. The two PD groups were impaired in rate of generation and release of isometric force but did not differ from each other.

$z=3.71, p<0.001)$. The two PD groups did not differ significantly from each other on either measure. These results show that isometric force generation and release are both abnormal in PD, in terms of rate of change of force as well as latency to respond; the treated and untreated patients did not differ on these measures.

CORRELATIONS WITH COGNITIVE, AFFECTIVE AND CLINICAL MOTOR DISABILITY

The homogeneity of findings in the two groups of patients with PD enabled ranked correlations to be performed on the whole PD group. Correlations were performed between the four experimental variables (latency and rate of force change for both generation and release) and scores on the KCRS, FFM, BDI, BDS, $M Q$, and the WCST (table 2).

No significant correlations were found in the PD group between the latency to force onset or release and any of the motoric, cognitive or affective scores. By contrast, the rate of force generation and the rate of force release correlated with both clinical motor measures, KCRS and FFM, and severity of depression. No correlation was found between any of the experimental variables and overall cognitive score as judged by the BDS or frontal lobe function as assessed by the WCST. However, significant correlations were found between both rate of force generation and release and memory quotient (for force generation, rho $=0.44, \quad \mathrm{p}<0.05 ;$ for force release rho $=0.39, \mathrm{p}<0.05)$.

\section{LONGITUDINAL STUDY (FIGURE 4)}

Compared with their first assessment, the control group showed no significant improvement on their second assessment on any of the measures of reaction time or rate of change of force. Thus controls showed no significant practice effects. Both the clinical motor measures, KCRS and FFM, improved in the subgroup of PD patients who were followed longitudinally, before and after treatment (for KCRS, $z=2 \cdot 77, p<0.01$; for FFM, $z=3 \cdot 21$, $\mathrm{p}<0.005)$. This PD subgroup also showed a significant improvement in the rate of release of force $(z=2.58, p<0.01)$ and a similar, though not statistically significant, trend for improvement in rate of force generation $(\mathrm{z}=1 \cdot 59, \mathrm{p}=0.112)$. There was, however, no significant change in the latency to force onset 
Table 2 Correlations (rho) of latency and rate of force change with measures of cognitive, affective and motor disability

\begin{tabular}{|c|c|c|c|c|}
\hline & \multicolumn{2}{|l|}{ Latency } & \multicolumn{2}{|c|}{ Force Change } \\
\hline & Onset & Release & Generation & Release \\
\hline $\begin{array}{l}\text { BDS } \\
\text { BDI } \\
\text { MQ } \\
\text { WCST categories } \\
\text { Fine finger movements } \\
\text { KCRS score }\end{array}$ & $\begin{array}{r}0.21 \\
0.35 \\
-0.35 \\
-0.01 \\
-0.23 \\
0.07\end{array}$ & $\begin{array}{r}0.20 \\
0.04 \\
-0.36 \\
-0.03 \\
-0.24 \\
-0.03\end{array}$ & $\begin{array}{l}-0 \cdot 18 \\
-0.48^{\star} \\
0 \cdot 45^{\star} \\
-0 \cdot 26 \\
0 \cdot 49^{\star \star} \\
-0.45^{\star}\end{array}$ & $\begin{array}{l}-0.01 \\
-0.50^{\star} \\
0.40^{\star} \\
-0.20 \\
0.41^{\star} \\
-0.11\end{array}$ \\
\hline
\end{tabular}

or release. No significant differences were observed related to the nature of treatment, but the numbers involved in each treatment group are small and genuine differences may be masked.

In summary, effective treatment of clinical motor disability in the PD patients was associated with a dissociation between the isometric force measures; latencies were unresponsive to treatment, but rate of force release showed a significant improvement.

\section{Discussion}

The results of this study unequivocally demonstrate an abnormal isometric force profile in PD. The profile was abnormal in terms of latency and rate of generation and relaxation of force; however, patients did achieve normal levels of maximal force so that abnormal force profile is not a non-specific effect of weakness. Positive correlations were demonstrated between the isometric force measures of rate of production and release of force and clinical motor disability, finger dexterity, severity of depression and memory quotient. No such correlations were seen for isometric latency measures. These results suggest that components of isometric force are dissociable in PD. This conclusion is supported by the effects of treatment: in concert with improved motor disability, treatment improved rate of force
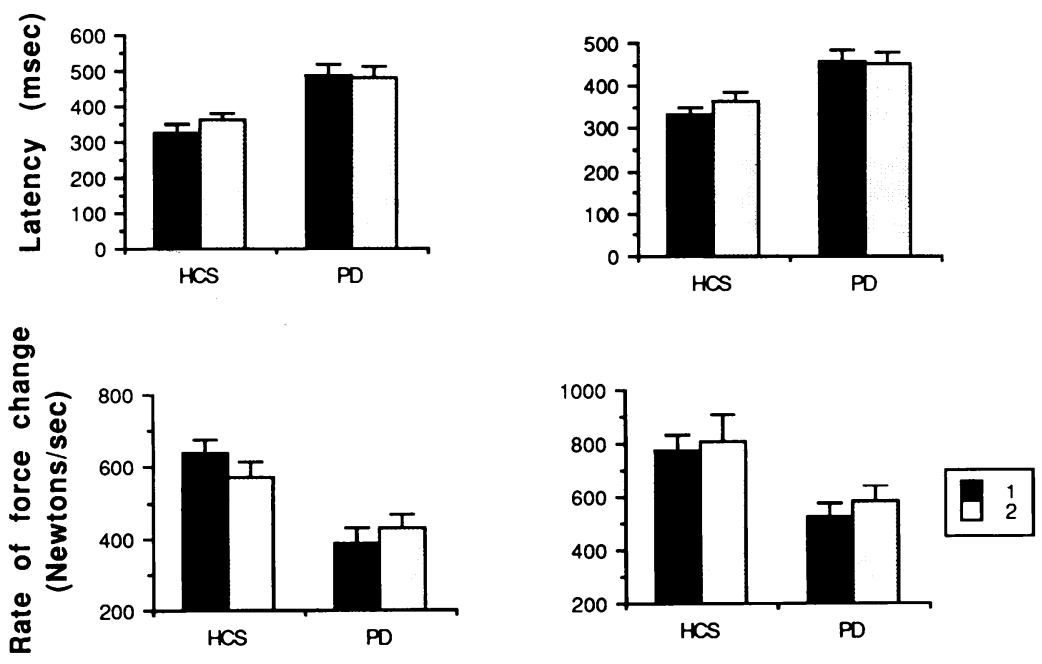

Figure 4 Latency and rate of change of force during onset and release of isometric force for de novo PD patients studied before (1) and after (2) treatment that produced significant improvement in clinical motor disability. Healthy control subjects were assessed on two occasions, separated by a similar interval. Results are expressed as mean and SEM. Treatment improved rate of force change but not latency of response. release and tended to improve rate of force generation but had no effect on response latency. These results add to our understanding of the nature of the motor deficits in $\mathrm{PD}$, specifically by accounting for the effects of treatment, disease duration and cognitive and affective disturbances. Moreover, the use of an isometric paradigm extends previous observations on isotonic movement but avoids many of the confounding variables, such as visual perception, spatial orientation, estimation and prediction.

Patients with PD were shown not to be weak but were unable to initiate force normally, as shown by the prolonged latency to force onset. This finding agrees with previous studies of isotonic movement in PD that demonstrate a slowing of movement onset times. ${ }^{14-16}$ However, isotonic and isometric reaction times may not be directly comparable. In an isotonic task, some limb movement is required, for example to release one button to hit a second, to turn a lever or even simply to release or leave a home key. Those processes involved in movement preparation, distance estimation, spatial location of the target and selection of the appropriate forces required to effect movement are all important aspects of success in any isotonic task. The isometric tasks in our experiment did not require overt limb movement, the location of a target, or estimation of distance and appropriate force level. Thus the tasks used may be less dependent on higher cognitive function and represent a more pure motor measure.

The finding of slowed force initiation and generation is in keeping with other studies of isometric force production in PD. ${ }^{17}{ }^{18}$ However, the study of Stelmach et al (1989) involved chronically-medicated, weak patients and examined reaction time and rate of force development to various fixed percentages of peak force. Our study extends these observations to newly-diagnosed, untreated patients of normal strength who were required to develop maximal force. The use of maximal force as an end-point in our study highlights the slowed rate of force generation in this group, even when the isometric equivalent of an isotonic ballistic movement is used.

The abnormalities in rate of force production are compatible with observations of EMG activity during movement in PD. Patients with $P D$ are unable to generate an adequately scaled EMG triphasic burst to carry out a successful movement. ${ }^{19}$ As a consequence, they employ a series of small amplitude bursts to complete the movement. In our experiment, patients reached normal levels of maximal force but appeared unable to achieve normal force production characteristics, despite maximal efforts.

The small amplitude EMG bursts seen during movement have been used to formulate a hypothesis that patients with PD have an inability to allocate appropriate "neural energy" into motor programmes that leads to akinesia. ${ }^{20}$ This hypothesis would predict that abnormalities of force profile would be greater in situations requiring greater effort, in partic- 
ular during force generation as opposed to force release, where effort is minimal. However, we have shown equivalent slowing during force release and generation which argues strongly against this hypothesis. Similar results were obtained by Wing (1988). Further work is necessary to determine the origin of this unexpected finding. A possible explanation includes a role of active processes in relaxation, that is, if contraction of antagonists is required for active relaxation then the "neural energy" hypothesis would still stand. Dyssynergia between agonists and antagonists may also explain the results in part. The positive correlations between rate of force release and clinical measures of akinesia and rigidity, but not tremor, also suggest that passive rigidity and failure to relax from active movement share a common pathophysiological basis. The interpretation is supported by the effects of treatment which produced a significant improvement in rate of relaxation of force as well as clinical motor disability. The findings support the suggestion that isometric tests provide the best measures of akinesia ${ }^{5}$ but extend the concept to relaxation as well as generation of force.

Rate of force generation and release correlated with clinical motor disability and severity of depression but not overall cognitive capacity or frontal lobe function. By contrast, latency to onset or release did not correlate with cognitive, clinical motor or affective variables. The results suggest dissociations between components of akinesia, notably between latencies and rates of change of force; the clinical and experimental measures of akinesia also correlate with depression (psychomotor retardation). Latency to isometric movement and psychomotor retardation may be based on separate neurochemical pathologies.

The results of the longitudinal study indicate a selective effect of the treatment on rates of change of force, latencies being relatively unaffected. This finding adds further weight to the dissociation between latencies and rates of change of force. Furthermore, it suggests that psychomotor retardation, which includes rates of change of force, is based upon nigrostriatal dopamine deficiency whereas latency to isometric movement may be more related to other neurochemical deficits, such as noradrenaline. In conclusion, we suggest that future research be directed at identification of the neurochem- ical bases of these dissociable processes of isometric motor control.

This research was supported by grants from Roche Products, UK and the Parkinson's Disease Society, UK. The author thank Dr GAB Davies-Jones, J Gumpert and G Venables fo referring patients for study, $\mathrm{Dr} \mathrm{N}$ Harvey for provision of some of the Beck scores on the PD group and Dr E Sullivan for useful discussion.

The results of this study were presented at the meeting of the American Academy of Neurology, Miami, Florida, April 1990. 1 Brown RG, Marsden CD. Neuropsychology and cognitive
function in Parkinson's disease: an overview. In: Marsden $\mathrm{CD}, \mathrm{Fahn}$ S. eds. Movement disorders 2. London: Butterworth 1987:99-123.

2 Sagar HJ, Sullivan EV. Patterns of cognitive impairment in dementia. In: Kennard C, ed. Recent advances in clinical neurology, Vol 5. Edinburgh: Churchill Livingstone neurology,
1988:47-86.

3 Bloxham CA, Mindell TA, Frith CD. Initiation and execution of predictable and unpredicatable movements in tion of predictable and unpredicatable mo
Parkinson's disease. Brain 1984;107:371-84.

4 Benecke R, Rothwell JC, Dick JPR, Day BL, Marsden CD. Performance of simultaneous movements in patients with Parkinson's disease. Brain 1986;109:739-57.

5 Benecke R, Rothwell JC, Dick JPR, Day BL, Marsden CD Disturbance of sequential movements in patients with Parkinson's disease. Brain 1987;100:361-79.

6 Sagar HJ, Jordan N, Cooper J, Sullivan EV. The dissociation between cognition and motor control in Parkinson' disease (Abstract). Neurology 1990;40 (Sup 1):168.

7 Blessed G, Tomlionson BE, Roth M. The association between quantitative measures of dementia and of senile changes in central grey matter of elderly subjects. $\mathrm{Br} f$ Psychiatry 1968;114:797-811.

8 Nelson HE, O'Connell A. Dementia: the estimation of premorbid intelligence level using the New Adult Reading premorbid intelligence level usi

9 Wechsler D, Stone CP. Wechsler Memory Scale. New York: Psychological Corporation, 1945.

10 Milner B. Effects of different brain lesions on card sorting: the role of the frontal lobes. Res Publ Ass Res Nerve Ment Dis 1958;36:244-57.

11 Beck AT, Ward CH, Mendelson M, Mock J, Erbaugh J. An inventory for measuring depression. Arch Gen Psychiatry 1961;4:561-71.

12 Brown RG, Marsden CD, Quin N et al. Alterations in affectarousal state during fluctuations in motor function in Parkinson's diseae. $\mathcal{F}$ Neurol Neurosurg Psychiatry 1984 47:454-65.

13 Heliwell $\mathrm{P}$, Howe A, Wright V. Functional assessment of the hand: reproducibility, acceptability and utility of a new system for measuring strength. Ann Rheumatic Dis 1987;46:203-8.

14 Angel RW, Alston W, Higgins JR. Control of movement in Parkinson's disease. Brain 1970;93:1-14.

15 Evarts EV, Teravainen H, Calne DB. Reaction time in Parkinson's disease. Brain 198;104:167-83.

16 Brown RG, Marsden CD. Visuospatial function in Parkinson's disease. Brain 1986;109:987-1002.

17 Stelmach GE, Worringham CJ. The preparation and production of isometric force in Parkinson's disease. Neuropsychologia 1987;26:93-103.

18 Stelmach GE, Teesdale N, Phillips J, Worringham CJ. Force production characteristics in Parkinson's disease. Exp Brain Res 1989;76:165-72.

19 Beradelli A, Dick JPR, Rothwell JC, Day BL, Marsden CD. Scaling the size of the first agonist EMG burst during rapid wrist movement in patients with Parkinson's disease. F Neurol Neurosurg Psychiatry 1986;49:1273-9.

20 Hallett M, Khoshbin S. A physiological mechanism of bradykinesia. Brain 1980;103:301-14.

21 Wing AM. A comparison of the rate of pinch grip force increase and decreases in parkinsonian bradykinesia. Neuropsychologia 1988;26:479-82. 